\title{
Review: tricyclic related drugs, but not classical tricyclics, are a tolerable alternative to SSRIs in older people with depression
}

Wilson K, Mottram P. A comparison of side effects of selective serotonin reuptake inhibitors and tricyclic antidepressants in older depressed patients: a meta-analysis. Int J Geriatr Psychiatry 2004;19:754-62.

\section{Q How do tricyclic antidepressants (TCAs) and TCA related drugs compare with selective serotonin reuptake inhibitors in terms of tolerability in older people with depression?}

\section{METHODS}

$\square$

Design: Systematic review with meta-analysis

Data sources: PsycINFO, MEDLINE, EMBASE, CINAHL, SINGLE, National Research Register, Dissertation Abstracts International, Biological Abstracts, Cochrane Controlled Trials Register, and the Cochrane Collaboration Depression, Anxiety and Neurosis

Controlled Trials, searched up to 2002, plus hand searches and contact with authors.

(1) Study selection and analysis: Randomised controlled trials (with concealment) comparing selective serotonin reuptake inhibitors (SSRIs) with TCAs in people aged over 60 years with depression. Trials were excluded if participants had other mental disorders or were receiving psychotherapy, except where data on psychotherapy and antidepressants were analysed separately. Two reviewers independently assessed trials, and included studies were meta-analysed for withdrawal rates and adverse effects. Random effects models were used for estimating effect size. Descriptive data were also extracted.

Outcomes: Total withdrawal rates; withdrawals due to adverse effects.

\section{MAIN RESULTS}

Eleven out of 37 identified trials met inclusion criteria (537 people receiving TCAs and 554 receiving SSRIs). Seven RCTs compared classical TCAs with SSRIs; the remaining four RCTs compared TCA related drugs. SSRIs significantly reduced total withdrawal rate and withdrawals due to adverse events compared with TCAs and TCA related drugs (see table). However, subgroup analyses by TCA class found that although classical TCAs significantly increased withdrawal compared with SSRIs, there was no significant difference between TCA related drugs and SSRIs.

Table Relative risks of withdrawal from treatment

\begin{tabular}{|c|c|c|}
\hline Comparison & $\begin{array}{l}\text { Relative risk of } \\
\text { withdrawal for any } \\
\text { reason }(95 \% \mathrm{Cl})\end{array}$ & $\begin{array}{l}\text { Relative risk of withdrawal } \\
\text { due to adverse effects } \\
(95 \% \mathrm{Cl})\end{array}$ \\
\hline All TCAs $v$ SSRIs & 1.24 (1.04 to 1.47$)$ & 1.30 (1.02 to 1.64$)$ \\
\hline Classical TCA $v$ SSRIs & $1.26(1.04$ to 1.52$)$ & 1.33 (1.04 to 1.71$)$ \\
\hline TCA related $v$ SSRIs & 1.49 (0.79 to 2.98$)$ & $1.07(0.43$ to 2.70$)$ \\
\hline
\end{tabular}

For correspondence: Professor Kenneth Wilson Professor of Old Age Psychiatry, University of Liverpool, Derby Road, St Catherine's Hospital, Birkenhead, Wirral CH42 OLQ, UK; kw500505@liverpool.ac.uk

Sources of funding: not stated.

\section{CONCLUSIONS}

TCA related drugs, but not classical TCAs, are as well tolerated as SSRIs in older people, and may be used to treat depression in this age group where intolerance to SSRIs develops.

\section{NOTES}

Classical TCAs include doxepin, amitriptyline, imipramine, clomipramine, dothiepin, nortriptyline, trimipramine, desipramine, and nomifensine. TCA related drugs include mianserin, trazadone, maprotoline, viloxazine. SSRIs include paroxetine, fluoxetine, citalopram, fluvoxamine, and sertraline.

Commentary

D epression in the elderly is a complex and challenging area. Its prevalence is difficult to establish, as the standard diagnostic criterion (DSM-IV) may not be applicable in older people, ${ }^{1}$ and the presence of multiple medical comorbidities complicates drug treatment choices. ${ }^{2}$ Older people with depression may also suffer more frequent relapses and require longer courses of treatment. ${ }^{3}$ Therefore the choice of an antidepressant is dictated by a number of factors that include efficacy, tolerability, and safety. On these grounds selective serotonin reuptake inhibitors (SSRIs) have become the first line agents for the pharmacological treatment of depression in the elderly.

However SSRIs are now well recognised to cause adverse reactions in a number of people which may include gastrointestinal tract disturbance, dizziness, ${ }^{4}$ postural hypotension, hyponatraemia, ${ }^{5}$ and the serotonergic syndrome. In addition, as with all antidepressants, not all patients will respond. What then is the next choice of agent?

This review compares side effect and withdrawal rates from a number of trials and concludes that tricyclic related antidepressants (TCA related) may offer an equally well tolerated alternative to SSRIs. Unfortunately, as the authors note, only a very small number of people with depression received TCA related agents and only two agents were studied (mianserin and trazadone). Newer agents such as venlefaxine, reboxetine, and mirtazipine have become available since many of the trials reviewed by the authors were published.

Classical tricyclics no longer have a role in the initial treatment of depression in the elderly except in unusual circumstances and a review such as Wilson and Mottram's confirms this. However, the choice of agent if an SSRI is not tolerated is still not clear and will need to be further studied. TCA related drugs are one option but until comparison with newer agents has occurred no clear recommendations can be made. Rohan Wee MBBS, FRACP

Geriatrician, Bundoora Extended Care Centre, Bundoora, Victoria, Australia

1 Burke WJ, Wengel SP. Late-life mood disorders. Clin Geriatr Med 2003; 19:777-97

2 Flint A. Pharmacologic treatment of depression in late life. Can Med Assoc J 1997; 157:1061-7.

3 Hybels CF, Blazer DG. Epidemiology of late-life mental disorders. Clin Geriatr Med 2003;19:663-96.

4 Whooly MA, Simon GE. Managing depression in medical outpatients. New Engl J Med 2000;343:1942-50.

5 Wee R, Lim WK. Selective serotonin re-uptake inhibitors (SSRIs) and hyponatraemia in the elderly. Int J Geriatr Psychiatry 2004;19:590-1. 